\title{
Newtonian and post-Newtonian approximations are asymptotic to general relativity
}

\author{
T. Futamase \\ Department of Applied Mathematics and Astronomy, University College, Cardiff, United Kingdom \\ Bernard F. Schutz \\ Max Planck Institut für Physik und Astrophysik, D-8046 Garching bei München, Federal Republic of Germany \\ and Department of Applied Mathematics and Astronomy, University College, Cardiff, United Kingdom*
}

(Received 18 March 1983)

\begin{abstract}
A precise definition of the Newtonian and post-Newtonian hierarchy of approximations to general relativity is given by studying a $C^{\infty}$ sequence of solutions to Einstein's equations that is defined by initial data having the Newtonian scaling property: $v^{i} \sim \epsilon, \rho \sim \epsilon^{2}, p \sim \epsilon^{4}$, where $\epsilon$ is the parameter along the sequence. We map one solution in the sequence to another by identifying them at constant spatial position $x^{i}$ and Newtonian dynamical time $\tau=\epsilon t$. This mapping defines a congruence parametrized by $\epsilon$, and the various post-Newtonian approximations emerge as derivatives of the relativistic solutions along this congruence. We thereby show for the first time that the approximations are genuine asymptotic approximations to general relativity. The proof is given in detail up to first post-Newtonian order, but is easily extended. The results will be applied in the following paper to radiation reaction in binary star systems, to give a proof of the validity of the "quadrupole formula" free from any divergences.
\end{abstract}

\section{INTRODUCTION}

Einstein ${ }^{1}$ built the Newtonian limit into general relativity, and every serious text on the subject contains a heuristic extraction of Newton's equations from Einstein's. Einstein also computed the first of the post-Newtonian effects, the precession of the perihelion, but a systematic attempt to build higher-order approximations in the Newtonian spirit was not made until the series of papers by Chandrasekhar and associates. ${ }^{2-4}$ Such a postNewtonian hierarchy of approximations is important in analyzing a number of relativistic problems, such as the orbits of close binary stars, ${ }^{5}$ solar-system tests of general relativity, ${ }^{6}$ and gravitational radiation reaction. ${ }^{4}$

The Newtonian limit may be thought of as two limits tied together. One limit is that the gravitational field gets weaker. The other limit is that all velocities and forces characteristic of the material system also get smaller, in order to permit the weakening gravity to remain an important effect on the system's dynamics. The limit is thus a limit involving two dynamical systems, matter and gravity: there is no pure vacuum Newtonian limit. Moreover, the time scale of interest in the limit is that of the material system-in the solar system, Earth years rather than the gravitational-wave crossing time. Mathematically, this means that in the limit one tries to preserve the hyperbolic form of the material system's dynamical equations. Relative to the material system's time scales, gravitationalwave propagation gets faster and faster, so that in the limit the hyperbolic field equations of general relativity go over to the elliptic field equation of Newton. This is a singular limit. What distinguishes this problem from the similarly singular slow-motion ("dipole") approximation to electromagnetism is the nonlinearity of the equations, a point we will return to in a moment.

Such a singular limit cannot be uniform everywhere for all time. Any binary pair of stars, no matter how weak its gravity and slow its orbital motion, will eventually spiral together due to gravitational radiation, and the effects of its Newtonian gravitational field will always be swamped by those of its gravitational waves far enough away along an outgoing null world line. Therefore, if the postNewtonian development is not done carefully, this singularity can lead to many formal problems, such as divergent integrals. Ehlers et al. ${ }^{7}$ catalogued the difficulties with the then-existing derivations and argued that, unless these difficulties were removed, the "quadrupole formula" prediction for radiation reaction in the binary pulsar system-now reasonably well confirmed by observations ${ }^{8}$ - could not be regarded as a firm consequence of general relativity. Since then, considerable improvements have been made in the post-Newtonian derivations, such as the work by Ehlers, ${ }^{9}$ Kerlick, ${ }^{10,11}$ and Walker and Will, ${ }^{12}$ but some divergent integrals remain and what seems to us the most fundamental question has not even been addressed: Is there some formulation of the post-Newtonian hierarchy which actually can be shown to provide an asymptotic approximation to general relativity? Our aim in this paper is to provide such a formulation. As a consequence of our having defined the problem so carefully, Futamase (following paper: paper II) will show that all the divergences of earlier schemes disappear in this one. He will therefore be able to apply this formulation to the question of radiation reaction to prove that the quadrupole formula does indeed give an asymptotic approximation to the behavior of relativistic systems.

The new feature of the present formulation is that it is based on the initial-value problem for gravity as well as matter. Most previous formulations have attempted to determine the dynamical degrees of freedom of the gravitational field by an asymptotic wave condition: either that gravitational waves were purely outgoing or that there was 
no incoming radiation. The mathematical difficulty with such wave conditions is that general relativity is nonlinear in such a way that the characteristics of the hyperbolic equations - the light cones-depend on the strength of the gravitational field and therefore upon the expansion parameter. As Ehlers $e t a l^{7}$ noted, it is very difficult to formulate a global radiation condition when the global behavior of the light cones is not known. On the other hand, one of us ${ }^{13}$ strongly criticized the use of such global conditions in the radiation-reaction problems, and showed that one could obtain the standard radiation-reaction formulas in linearized gravity (i.e., weak gravity but not weak material forces) and in electromagnetism from an initialvalue approach that made few global assumptions. The formulation of the Newtonian limit in this paper provides the framework for the extension of this point of view to Newtonian systems in paper II.

The history of this problem is so full of controversy and of rather subtle differences between various approaches that we have decided to make the logical development of this exposition relatively self-contained, so that the reader need not refer to earlier papers in order to understand the present one except for details of calculations. The reader should bear in mind that, in a chain of ideas going back to Einstein, the original contributions we claim here are (i) the initial-value approach, and (ii) the use of oneparameter sequences of solutions to Einstein's equations to show the asymptotic nature of the hierarchy of approximations. For comparisons among various previous approaches see Anderson and Decanio, ${ }^{14}$ Kerlick, ${ }^{10,11}$ and Walker and Will. ${ }^{12}$

\section{SCALING THE NEWTONIAN EQUATIONS}

Extracting the Newtonian equations from general relativity begins with an understanding of a scaling property of the Newtonian equations which permits one to change the time scale of any solution in a simple way. We shall concentrate on perfect fluids in this paper, although analogous results may be found for more realistic systems. The Newtonian equations involve the six variables, density $(\rho)$, pressure $(p)$, gravitational field $(\Phi)$, and velocity $\left(v^{i}\right.$, $i=1,2,3)$ :

$$
\begin{aligned}
& \nabla^{2} \Phi-4 \pi \rho=0, \\
& \partial_{t} \rho+\nabla_{i}\left(\rho v^{i}\right)=0, \\
& \rho \partial_{t} v^{i}+\rho v^{j} \nabla_{j} v^{i}+\nabla^{i} p+\rho \nabla^{i} \Phi=0, \\
& p-f(\rho)=0,
\end{aligned}
$$

where we have set $G=1$. Equation (2.4) is the equation of state.

\section{Newtonian scaling theorem}

If the set of functions $\left\{\rho\left(x^{i}, t\right), p\left(x^{i}, t\right), \Phi\left(x^{i}, t\right), v^{i}\left(x^{j}, t\right)\right\}$ satisfies Eqs. (2.1)-(2.3), then the replacements

$$
\begin{aligned}
& \rho \rightarrow \epsilon^{2} \rho\left(x^{j}, \epsilon t\right), \quad p \rightarrow \epsilon^{4} p\left(x^{j}, \epsilon t\right), \\
& \Phi \rightarrow \epsilon^{2} \Phi\left(x^{j}, \epsilon t\right), \quad v^{i} \rightarrow \epsilon v^{i}\left(x^{j}, \epsilon t\right)
\end{aligned}
$$

also satisfy the same equations.

The proof is trivial algebra. The scaling of the time variable means that the system for $\epsilon=1$ is doing at time $t$ what the system for any other $\epsilon$ is during at time $t / \epsilon$ : the weaker the gravity $(\epsilon \rightarrow 0)$ the longer the time scale.

Notice that the equation of state, Eq. (2.4), which is necessary for a well-determined set of equations, does not scale automatically, so that solutions of Newton's equations are scale invariant only if we write an explicit $\epsilon$ dependence into the equation of state as

$$
p-\epsilon^{4} f\left(\epsilon^{-1} \rho\right)=0 \text {. }
$$

Realistic equations of state do not scale this way, which simply means that, say, along the "main sequence" (i.e., the relation between luminosity and mass obeyed by ordinary stars) the density does not change as $\rho\left(x^{j}\right) \rightarrow \epsilon^{2} \rho\left(x^{j}\right)$, but rather the spatial distribution of density changes as well. Of course, it is possible to find simple equations of state that do scale. For example, an ideal gas obeys the equation $p=b \rho T$, where $b$ is a constant. Then if we take $T \rightarrow \epsilon^{2} T$, we will have (2.6). This is reasonable on grounds of kinetic theory: $T \sim\left\langle v^{2}\right\rangle$. A very familiar example is the polytrope, $p=K \rho^{1+1 / n}$, where the replacement $K \rightarrow \epsilon^{2-2 / n} K$ ensures the proper scaling. ${ }^{15}$

\section{REGULAR, ASYMPTOTICALLY NEWTONIAN SEQUENCES}

We wish to develop an asymptotic approximation to general relativity, asymptotic as the scaling variable $\epsilon$ approaches zero. It is an elementary observation, but one that has not been sufficiently stressed before in this context, that an asymptotic approximation is fundamentally an approximation to a function, not to the function's value for some $\epsilon$. That is, $g(\epsilon)$ approximates $f(\epsilon)$ to order $\epsilon^{p}$ if $|f(\epsilon)-g(\epsilon)| / \epsilon^{p}$ goes to zero as $\epsilon \rightarrow 0$. This definition uses $f$ in a neighborhood of $\epsilon=0$ and not just $f$ at one value of $\epsilon$. To approximate general relativity, therefore, we need a sequence of solutions of general relativity which has the Newtonian character as $\epsilon \rightarrow 0$.

Einstein's equations are nonlinear in the field variables, however, so it will not be possible to enforce the scaling of Eq. (2.5)—with $\Phi$ replaced by some suitably scaled metric-everywhere in spacetime. We shall therefore impose it only on the initial data for the solutions in the sequence. We shall see later that at later times $\rho$ will contain higher-order terms in $\epsilon$, but of course none of lower order than $\epsilon^{2}$ arise. The failure to allow explicitly higherorder terms in $\rho$ and $v^{i}$ characterizes most previous formulations of the post-Newtonian hierarchy.

The initial data must determine solutions of the following equations, which we write in the form used by Anderson and Decanio. ${ }^{14}$ We define the gravitational field vari$\mathrm{able}^{16}$

$$
\bar{h}^{\mu \nu}=\eta^{\mu \nu}-(-g)^{1 / 2} g^{\mu \nu},
$$

where $\eta^{\mu \nu}$ is the metric of Minkowski spacetime, and adopt the Lorentz (= harmonic, de Donder) gauge,

$$
\bar{h}^{\mu \nu}{ }_{, v}=0 \text {. }
$$

In this gauge the field equations are

$$
\eta^{\mu v} \bar{h}_{, \mu \nu}^{\alpha \beta}=-16 \pi \Lambda^{\alpha \beta},
$$

where 


$$
\begin{aligned}
& \Lambda^{\alpha \beta}=\theta^{\alpha \beta}+\chi^{\alpha \beta \mu v}{ }_{, \mu v}, \\
& \chi^{\alpha \beta \mu v}=(16 \pi)^{-1}\left(\bar{h}^{\alpha v} \bar{h}^{\beta \mu}-\bar{h}^{\alpha \beta} \bar{h}^{\mu v}\right), \\
& \theta^{\alpha \beta}=(-g)\left(T^{\alpha \beta}+t_{\mathrm{LL}}^{\alpha \beta}\right),
\end{aligned}
$$

and where $T^{\alpha \beta}$ is the perfect-fluid stress-energy tensor

$$
\begin{aligned}
& T^{\alpha \beta}=(\rho+p) u^{\alpha} u^{\beta}+p g^{\alpha \beta}, \\
& u^{\alpha} u^{\beta} g_{\alpha \beta}=-1,
\end{aligned}
$$

and $t_{\mathrm{LL}}^{\alpha \beta}$ is the Landau-Lifshitz pseudotensor. ${ }^{14,17}$ Equations (3.2) and (3.3) together imply the conservation law

$$
\Lambda_{, \beta}^{\alpha \beta}=0 \text {. }
$$

We shall take as our basic variables the set $\left\{\rho, p, v^{i}, \bar{h}^{\alpha \beta}\right\}$, with the definition

$$
v^{i}=u^{i} / u^{0}
$$

The final component of $u^{\alpha}$ can be found from Eq. (3.8). To make a well-determined system of equations we must add the conservation law for particles, whose number density $n$ is some function of $p$ and $\rho$ :

$$
\left(n u^{\alpha}\right)_{; \alpha}=0 \text {. }
$$

Equations (3.9) and (3.11) imply that the flow is adiabatic. The role of the equation of state is played by the arbitrary function $n(\rho, p)$.

The dynamical equations require initial data for $\bar{h}^{\mu \nu}, \bar{h}^{\mu \nu}{ }_{0,0}, \rho, p$, and $v^{i}$, but not all these data are independent. In particular, Eqs. (3.2) and (3.3) imply the four constraint equations

$$
\eta^{i j} \bar{h}^{\mu 0}{ }_{, i j}+16 \pi \Lambda^{\mu 0}-\eta^{i j} \bar{h}^{\mu}{ }_{i, j}{ }^{0}=0,
$$

which are relations only among the initial data. We shall take the point of view that one gives $\bar{h}^{i j}$ and $\bar{h}^{i j}{ }_{, 0}$ independently and then solves Eq. (3.12) for $\bar{h}^{\mu 0}(\mu=0, \ldots, 3)$ and Eq. (3.2) for $\bar{h}^{\mu 0}{ }_{, 0}$. Note that these solutions cannot be obtained explicitly, since $\Lambda^{\mu 0}$ contains $\bar{h}^{\mu 0}$, but they can be obtained iteratively in the manner we shall describe.

Our interest in this paper is to define the Newtonian hierarchy, not directly to study radiation reaction. Gravitational waves only complicate matters, so we will confine our attention here to the simplest situation, namely, where the initial data for $\bar{h}^{i j}$ and $\bar{h}^{i j}, 0$ are, zero. One can show ${ }^{18}$ that such initial data satisfy the Ó Murchadha and York $^{19}$ criterion for the absence of radiation far away from the matter. The case where there is more general radiation present initially will be considered in paper II in the context of the radiation-reaction problem. Accordingly we adopt the following definition.

Definition. A regular, asymptotically Newtonian sequence is a sequence of solutions of Einstein's equations for a perfect fluid defined by the following sequence of Cauchy data:

$$
\begin{aligned}
& \rho\left(t=0, x^{j}, \epsilon\right)=\epsilon^{2} a\left(x^{j}\right), \\
& p\left(t=0, x^{j}, \epsilon\right)=\epsilon^{4} b\left(x^{j}\right), \\
& v^{i}\left(t=0, x^{j}, \epsilon\right)=\epsilon c^{i}\left(x^{j}\right), \\
& \bar{h}^{i j}\left(t=0, x^{j}, \epsilon\right)=0, \\
& \bar{h}^{i j}{ }_{0}\left(t=0, x^{j}, \epsilon\right)=0,
\end{aligned}
$$

where the functions $a, b$, and $c^{i}$ are $C^{\infty}$ functions that have compact support contained entirely within a sphere of radius $R$ around the origin. If one wishes to relate $\rho$ and $p$ by an equation of state, then one should adopt the Newtonian scaling of it in Sec. II.

It may be helpful to visualize the sequence as a fiber bundle whose base space is the real line (parameter $\epsilon$ ) and whose fibers are diffeomorphic to $R^{4}$, each being spacetime ${ }^{20}$ for a particular value of $\epsilon$. The fiber $\epsilon=1$ may be a relativistic system we are particularly interested in. The fiber $\epsilon=0$ is Minkowksi spacetime. The use of the word "asymptotic" in the definition is justified by the theorem in Sec. IV below.

Of course, if, as we shall see, the material systems have a Newtonian behavior as $\epsilon \rightarrow 0$, then the dynamics will be slower and slower in this limit. As we stressed in Sec. I, we are interested in a map between fibers which preserves the dynamics of the material system, and such a map is obtained by defining the dynamical time

$$
\tau=\epsilon t
$$

As we saw in Sec. II, a map between fibers at constant $\tau$ and $x^{i}$ is natural to the problem: If the system for $\epsilon=0.1$ is a binary system which has completed its first orbit after a certain dynamical time $\tau_{1}$, then the system for $\epsilon=0.01$ will have completed its first orbit after roughly the same dynamical time $\tau_{1}$. (We say "roughly" rather than "exactly" because the system is not perfectly Newtonian.) The dynamical-time map is illustrated in Fig. 1. This map never reaches the fiber $\epsilon=0$; we shall see that in the limit $\epsilon=0$ the map defines the Newtonian and post-Newtonian approximations.

In order to do the calculations in the next section we must make some assumptions about regular, asymptotically Newtonian sequences. All of these are in principle provable, but proofs depend mainly upon a much better understanding than we now possess of the existence and uniqueness of solutions to the Cauchy problem for perfect fluids of compact support.

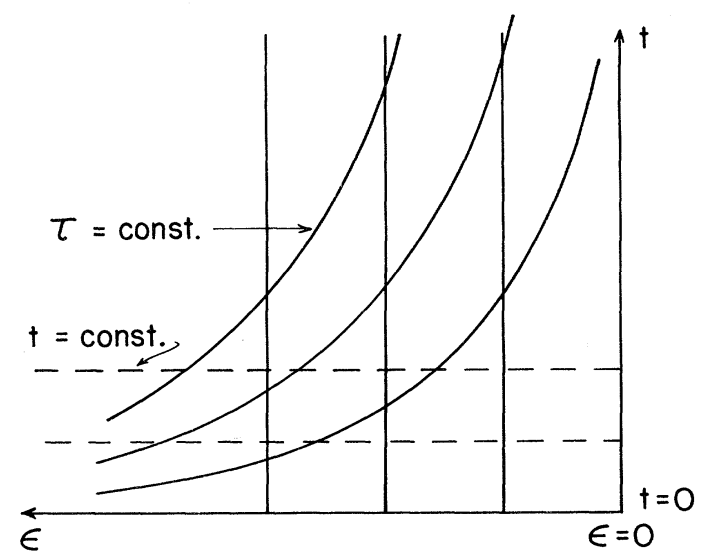

FIG. 1. Structure of the sequence of solutions. Vertical lines are the $t$ dimension of spacetime manifolds that solve Einstein's equations for initial data posed on the horizontal line $t=0$ on the bottom. Dashed horizontal lines are constant $t$. The dynamical-time map (see text) follows the hyperbolas. 
Assumptions. Recall that the initial data are $C^{\infty}$ functions of $x^{i}$ and $\epsilon$. We assume that regular, asymptotically Newtonian sequences have the following properties.

(i) The solutions for $t \neq 0$ are $C^{\infty}$ in $x^{i}, \epsilon$, and $\tau$ for $0<\epsilon<1,0 \leq \tau<\tau_{1}$, and all $x^{i}$. The important assumption here is that the validity of the solution in time is limited by some fixed dynamical time $\tau_{1}$ rather than a proper time $t_{1}$ for $\epsilon$ near zero.

(ii) We shall see that the different post-Newtonian approximations appear as $\epsilon \rightarrow 0$ limits of the derivatives of different orders with respect to $\epsilon$ of functions defined on the relativistic sequence. In our ignorance of the relativistic initial-value problem for this sort of system, we cannot prove that such limits exist. Instead, we will assume that these limits exist unless this assumption leads to contradictions. In other words, we shall derive relations among the limits and assume they exist until, for example, the relations show that the $(n+1)$ st derivative of a function cannot have a limit if its derivatives up to order $n$ do. In such a case we will assume that the first $n$ derivatives do exist and the next does not.

(iii) We require one technical assumption which makes it possible to calculate the approximations for $\epsilon=0$ without knowing details of the relativistic solutions for $\epsilon \neq 0$, which is that for any metric component or its $\epsilon$ derivative of any order, say $f\left(\tau, x^{j}, \epsilon\right)$, the $\epsilon \rightarrow 0$ limit is weakly uniform in the following sense when it exists: $\exists R, \alpha, \beta$ such that

$$
\lim _{\epsilon \rightarrow 0} f\left(\tau, x^{j}, \epsilon\right) / f\left(\tau, x^{j}, 0\right)=1+O\left(\epsilon^{\alpha}\right) O\left(r^{\beta}\right),
$$

for all $\left|x^{j}\right| \equiv r>R$.

$$
\alpha>\beta, \alpha>0
$$

(iv) Just as we need assumptions about the relativistic solutions, we also assume that the Newtonian and postNewtonian hierarchy are well-behaved initial-value problems, whose solutions are $C^{\infty}$ in $x^{j}$ and $\tau$ for $C^{\infty}$ initial data.

\section{THE POST-NEWTONIAN HIERARCHY}

Any $C^{n}$ function $f(\epsilon)$ whose $(n+1)$ st derivative exists has the asymptotic expansion about $\epsilon=0$ given by Taylor's theorem:

$$
\begin{aligned}
f(\epsilon)= & f(0)+\epsilon f^{\prime}(0)+\frac{\epsilon^{2}}{2} f^{\prime \prime}(0)+\cdots \\
& +\frac{\epsilon^{n}}{n !} f^{(n)}(0)+R_{n+1}
\end{aligned}
$$

where the remainder term $R_{n+1}$ is

$$
\begin{aligned}
R_{n+1} & =\frac{\epsilon^{n+1}}{(n+1) !} \int_{0}^{1}(1-l)^{n+1} \frac{d^{n+1}}{d \epsilon^{n+1}}(l \epsilon) d l \\
& =o\left(\epsilon^{n}\right) .
\end{aligned}
$$

If we want to obtain an asymptotic approximation to our sequence of relativistic systems, expressed as a sum of Newtonian, post-Newtonian, post-post-Newtonian, etc., contributions, then clearly we should try to identify these approximations with derivatives of the relativistic sequence with respect to $\epsilon$ at $\epsilon=0$. The key point is that the derivatives must be taken along the dynamical-time map, i.e., with $\tau$ fixed.

\section{Post-Newtonian hierarchy theorem}

Consider a regular, asymptotically Newtonian sequence with the properties defined and assumed in Sec. III. Let $\vec{\epsilon}$ be the vector field $d / d \epsilon$ along the congruence $\{\tau=$ const, $x^{i}=$ const $\}$ in the fiber bundle of the sequence. Then the Newtonian limit of the sequence is its fourth Lie derivative ${ }^{21}$ with respect to $\vec{\epsilon}$ at $\epsilon=0$, and the post-Newtonian correction is the sixth Lie derivative. The post-Newtonian correction so obtained is essentially equivalent to that obtained by earlier workers. ${ }^{2,10,14}$ Although there are subtle differences, which we shall point out, the sequence is at least $C^{7}$ in this sense at $\epsilon=0$, so the post-Newtonian approximation (up to sixth order) is asymptotic to the relativistic sequence as $\epsilon \rightarrow 0$.

Proof. The remainder of this section gives the main steps of the proof while omitting the considerable amount of straightforward algebra, much of which is of course identical to that of previous authors. Because of the complexity of the algebra we are stating and proving the theorem only to post-Newtonian order. Its extension beyond is straightforward and will be discussed in the next section and paper II. Because we take limits at fixed $\tau$, the material equations remain causal while, as discussed in Sec. I, the gravitational field equations become singular. We therefore follow Anderson and Decanio ${ }^{14}$ and express the gravitational field equations in the integral form given by Kirchhoff's formula ${ }^{22}$ :

$$
\begin{aligned}
\bar{h}^{\mu v}\left(\tau, x^{j}, \epsilon\right)= & 4 \int_{C\left(\tau, x^{j}, \epsilon\right)} \Lambda^{\mu v}\left(\tau-\epsilon r, y^{j}, \epsilon\right) r^{-1} d^{3} y+\frac{\tau}{4 \pi} \int_{S\left(\tau, x^{j}, \epsilon\right)} \bar{h}_{, \tau}^{\mu v}\left(\tau=0, y^{j}, \epsilon\right) d \Omega_{y} \\
& +\frac{1}{4 \pi} \frac{\partial}{\partial \tau}\left[\tau \oint_{S\left(\tau, x^{j}, \epsilon\right)} \bar{h}^{\mu v}\left(\tau=0, y^{j}, \epsilon\right) d \Omega_{y}\right],
\end{aligned}
$$

where

$$
r=\left|y^{j}-x^{j}\right|
$$

and $C\left(\tau, x^{j}, \epsilon\right)$ is the past flat-space light cone of the event $\left\{\tau, x^{j}\right\}$ in the spacetime given by $\epsilon$, truncated where it intersects the initial hypersurface $\tau=0$-see Fig. 2-on the sphere $S\left(\tau, x^{j}, \epsilon\right)$. Equation (4.3) expresses $\bar{h}^{\mu \nu}$ as a func- tional of its initial data (angular integrals over $S$ ) and its source $\Lambda^{\mu \nu}$. This is not an explicit expression, of course, since $\Lambda^{\mu v}$ contains $\bar{h}^{\mu \nu}$, but in the iteration it will always give the $n$th derivative of $\bar{h}^{\mu \nu}$ with respect to $\epsilon$ in terms of derivatives of order less than $n$ only. The use of the retarded integrand $\Lambda^{\mu \nu}\left(\tau-\epsilon r, y^{j}, \epsilon\right)$ does not reflect some arbitrary choice of retarded versus advanced interactions: it arises simply because we are asking for $\bar{h}^{\mu \nu}$ for $\tau>0$. 


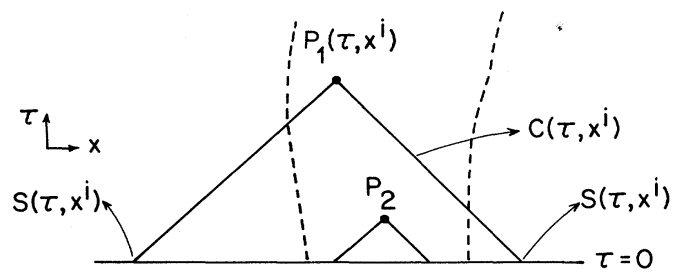

FIG. 2. The solution of the wave equation at any event $\left(\tau, x^{i}\right)$ for fixed $\epsilon$ has two pieces: a homogeneous solution which evolves from data at $\tau=0$, and an inhomogeneous solution which is an integral over the past light cone $C\left(\tau, x^{i}\right)$ truncated at $\tau=0$. The dashed lines enclose the support of the fluid. If the integrand of the retarded integral has compact support, then the truncated retarded integral for $P_{1}$ is equivalent to the usual fully retarded integral, but that for $P_{2}$ is not.

Equation (4.3) is the unique formal solution to Eq. (3.3) for given initial data.

Notice that the domains of integration are finite as long as $\epsilon \neq 0$, their diameter increasing as $\epsilon^{-1}$ as $\epsilon \rightarrow 0$. This linkage of the region of integration with the "weakness" parameter $\epsilon$ turns out to be crucial in eliminating the divergences that others have encountered.

Equations (4.3), (3.9), and (3.11) are the starting point for the construction of the approximations. The Lie derivatives of our theorem are simply partial derivatives with respect to $\epsilon$ holding fixed the coordinates $\tau$ and $x^{j}$, provided we express tensor indices with respect to $\tau$ rather than $t$. So from now on we shall deal with, for example, $T^{\tau \tau}=\epsilon^{2} T^{t t}$, which we shall see is of order $\epsilon^{4}$. Similarly the other components $T^{\tau i}$ and $T^{i j}$ are $O\left(\epsilon^{4}\right)$ as well. We shall adopt the notation

$$
{ }_{n} f\left(\tau, x^{i}\right)=\frac{1}{n !} \lim _{\epsilon \rightarrow 0}\left[\frac{\partial}{\partial \epsilon}\right]^{n} f\left(\tau, x^{i}, \epsilon\right) .
$$

Before developing the approximations, it is useful to prove three lemmas about the behavior of terms in Eq. (4.3) as $\epsilon \rightarrow 0$.

Lemma 1. Let a function $g\left(x^{j}, \epsilon\right)$ in the hypersurface $\tau=0$ he $C^{\infty}$ in $\epsilon$ at $\epsilon=0$, with its first $N \geq 0$ derivatives vanishing there, and let $\left|x^{j}\right|^{-M} g\left(x^{j}, \epsilon\right)$ be $C^{\infty}$ in $x^{j}$ at $\left|x^{j}\right|=\infty$ for some $M \leq N$. Consider the angular integral over the solid-angle element $d \Omega_{y}$ :

$$
J\left(\tau, x^{j}, \epsilon\right)=\oint_{S\left(\tau, x^{j}, \epsilon\right)} g\left(y^{j}, \epsilon\right) d \Omega_{y},
$$

where $S$ is the sphere introduced in Eq. (4.3). Then ${ }_{k} J\left(\tau, x^{j}\right)$ exists for $k \geq 0$, vanishes for $k<N-M$, and has contributions from ${ }_{n} g\left(x^{j}\right)$ for $N \leq n \leq M+k$. [The explicit expression is Eq. (4.7) below.]

Proof of Lemma 1. Write the integrand $g\left(y^{j}, \epsilon\right)$ as $g\left(r^{j}+x^{j}, \epsilon\right)$, where $r^{j}=y^{j}-x^{j}$ is the radius from the center of the sphere $S$, so that $\left|r^{j}\right|=r=\tau / \epsilon$. Writing $g$ as a function of spherical coordinates, we define a function $h$ by $g\left(r^{j}+x^{j}, \epsilon\right)=h\left(x^{j}, \epsilon, r, \Omega\right)$, where $\Omega$ stands for the angular dependence. This is integrated out, giving

$$
J\left(\tau, x^{j}, \epsilon\right)=\oint h\left(x^{j}, \epsilon, \tau / \epsilon, \Omega\right) d \Omega .
$$

The $\epsilon$ dependence of $J$ is now entirely in its integrand, and the conditions of the lemma guarantee it is regular: Although $\epsilon \rightarrow 0$ sends $r=\tau / \epsilon$ to $\infty$, causing $h$ to blow up as $r^{M}$, the explicit $\epsilon$ dependence of $h$ causes it to decay as $\epsilon^{N}$, giving a net $\epsilon^{N-M}$ behavior. Some algebra gives for $k>N-M$

$$
{ }_{k} J\left(\tau, x^{j}\right)=\sum_{m=0}^{k+M-N} \frac{1}{\tau^{M-m} m !(k-m+M) !} \oint d \Omega\left[\frac{\partial^{k-m+M}}{\partial \epsilon^{k-m+M}} \frac{\partial^{m}}{\partial \eta^{m}}\left(\eta^{M} h\left(x^{j}, \epsilon, \eta^{-1}, \Omega\right)\right)\right]_{\substack{\epsilon=0 \\ \eta=0}}
$$

and ${ }_{k} J\left(\tau, x^{j}\right)=0$ for other non-negative $k$. (In this equation, $\eta$ is just $r^{-1}$.) This completes the proof of the lemma.

Lemma 2. Consider the retarded integral

$$
I\left(\tau, x^{j}, \epsilon\right)=\int_{C\left(\tau, x^{j}, \epsilon\right)} f\left(\tau-\epsilon r, y^{j}, \epsilon\right) d r d \Omega_{y},
$$

where $C$ is the truncated past light cone of $\left(\tau, x^{j}\right)$ as in Eq. (4.3), and $r$ is the radius $\left|y^{j}-x^{j}\right|$. (i) Unless special assumptions about $f$ are made, the limit as $\epsilon \rightarrow 0$ of $I$ is the retarded integral

$I\left(\tau, x^{j}, 0\right)=\lim _{\epsilon \rightarrow 0} \epsilon^{-1} \int_{0}^{\tau} \int_{4 \pi} f\left(\tau-\xi, x^{j}+\xi n^{j} / \epsilon, 0\right) d \xi d \eta_{n}$,

where $n^{j}$ is a radial unit vector and $d \Omega_{n}$ integrates it over the unit sphere. (ii) Suppose

$$
\left|f_{, \tau}\left(\tau_{1}, x^{j}, 0\right)\right| \leq A\left|x^{j}\right|^{-1}\left|f\left(\tau_{2}, x^{j}, 0\right)\right|
$$

for $\left|x^{j}\right|>R, A$ and $R$ some constants, and $\tau_{1}, \tau_{2}$ arbitrary. Suppose also that $I\left(\tau, x^{j}, 0\right)$ is not "unusually" small, i.e., that $\lim _{\epsilon \rightarrow 0}\left|I / \int\right| f\left|d r d \Omega_{y}\right|$ is not zero. Then we need not retard the integral in (4.9):
$I\left(\tau, x^{j}, 0\right)=\lim _{\epsilon \rightarrow 0} \epsilon^{-1} \int_{0}^{\tau} \int_{4 \pi} f\left(\tau, x^{j}+\xi n^{j} / \epsilon, 0\right) d \xi d \Omega_{n}$.

Proof. The algebra is straightforward, replacing $r$ by $\xi / \epsilon$ in (4.8) to get (4.9). We need assumption (iii) of Sec. III to set the final $\epsilon$ to zero inside $f$ in (4.9). To prove (ii) we suppress the dependence of $f$ on $x^{j}$ and $n^{j}$ and write

$$
f\left(\tau-\xi, x^{j}+\xi n^{j} / \epsilon, 0\right)=h(\tau-\xi, \xi / \epsilon) .
$$

Then we have

$$
\begin{aligned}
h(\tau-\xi, \xi / \epsilon)=h(\tau, \xi / \epsilon) & -\int_{0}^{\xi} h_{, \tau}(\tau-\eta, \xi / \epsilon) d \eta \\
\int_{0}^{\tau} h(\tau-\xi, \xi / \epsilon) d \xi= & \int_{0}^{\tau} h(\tau, \xi / \epsilon) d \xi \\
& -\int_{0}^{\tau} \int_{0}^{\xi} h_{, \tau}(\tau-\eta, \xi / \epsilon) d \eta d \xi
\end{aligned}
$$

Denote the second term by $I_{1}$. Then we have

$$
\begin{aligned}
& I_{1}=\int_{0}^{\epsilon R} \int_{0}^{\xi} h_{, \tau}(\tau-\eta, \xi / \epsilon) d \eta d \xi+I_{2}, \\
& I_{2}=\int_{\epsilon R}^{\tau} \int_{0}^{\xi} h_{, \tau}(\tau-\eta, \xi / \epsilon) d \eta d \xi .
\end{aligned}
$$

As $\epsilon \rightarrow 0, I_{1} \rightarrow I_{2}$ as long as $h_{, \tau}$ is bounded. Then the limit of $I_{2}$ is bounded by 


$$
\left|I_{2}\right| \leq A \epsilon \int_{\epsilon R}^{\tau} \xi^{-1} \int_{0}^{\xi}|h(\tau-\eta, \xi / \epsilon)| d \eta d \xi .
$$

By the mean-value theorem there is some $\eta_{0}, 0 \leq \eta_{0}<\xi$, for which

$$
\left|I_{2}\right| \leq A \epsilon \int_{\epsilon R}^{\tau}\left|h\left(\tau-\eta_{0}, \xi / \epsilon\right)\right| d \xi
$$

and this makes a vanishingly small contribution compared to the term that survives in Eq. (4.10). This proves the lemma.

Remark 1. The fact that one cannot in general ignore retardation even in the limit $\epsilon \rightarrow 0$ is crucial: It will be shown in paper II that ignoring this fact leads to some of the divergent integrals at high order found by Kerlick ${ }^{11}$ and others. Although the integrand in Eq. (4.8) contains $\tau-\epsilon r$, this does not go over to $\tau$ in the limit, since the range of integration expands in such a way that $\tau-\epsilon r$ always ranges from $\tau$ to 0 . Earlier authors all expanded the integrand in (4.8) about $\tau$ :

$$
f(\tau-\epsilon r, \ldots)=f(\tau, \ldots)-\epsilon r f_{, \tau}(\tau, \ldots)+\cdots
$$

and assigned the second term to a higher order because of its explicit $\epsilon$ in front. This is incorrect in our scheme, because $\epsilon r$ is not uniformly small. If we were to factor $\epsilon$ out and integrate, say, $r f_{, \tau}(\tau, \ldots)$ over $C\left(\tau, x^{j}, \epsilon\right)$, we would in general find it diverging as $\epsilon^{-1}$ relative to that of $f(\tau, \ldots)$, because the integral of $\epsilon r f_{, \tau}$ is in fact of the same order as that of $f$ itself. This is indeed the character of Kerlick's most strongly divergent integrals. Only if the integrand has some special property, as in part (ii) of the lemma, can retardation be ignored. This happens in the lower-order post-Newtonian terms, as we shall see, and makes the formal slow-motion approximation sensible. But at some point the nonlinearity of Einstein's equations creates terms which do not satisfy (ii), and then retardation may be necessary at that order.
Why should some integrands obey (ii)? Not because of the slow-motion assumption: that relates to the $\epsilon$ behavior, not the $r$ behavior. Rather, it occurs because of conservation laws. For example, the Newtonian gravitational field has the asymptotic behavior $\Phi \sim M r^{-1}+D r^{-2}+Q r^{-3}+\cdots$, but conservation of mass and momentum ensure that $M_{, t}=D_{, t}=0$, so that $\Phi_{, t} \sim r^{-3}$. Conversely, an integrand fails to satisfy the conditions of part (ii) when its leading terms are dictated by time-dependent quantities, like the quadrupole moment, and this is where gravitational waves can be expected. So when waves become sources of higher-order corrections, retardation cannot be ignored, and the formal "slow-motion" iteration procedure-which neglects all retardation effects at any order-breaks down.

Remark 2. The criterion given in condition (ii) of Lemma 2 is sufficient for neglecting retardation, but not necessary. In fact, in paper II weaker conditions will be shown to suffice in particular cases. We believe, in fact, that condition (ii) could be replaced by the much weaker requirement that if

$$
\int \xi f_{, \tau}\left(\tau-\xi, x^{j}+\xi n^{j} / \epsilon, 0\right) d \xi d \Omega
$$

exists in the limit $\epsilon \rightarrow 0$, then retardation may be neglected in Eq. (4.9). We have no general proof of this, but in the Appendix we give arguments we find compelling.

The retarded integral may be differentiated in the following circumstances.

Lemma 3. Assume the integral $I$ of Eq. (4.8) exists. Suppose $f\left(\tau, y^{j}, \epsilon\right)\left|y^{j}\right|^{2}$ is $C^{\infty}$ in $r$ at $r=\infty, C^{1}$ in $\tau$, and $C^{1}$ in $\epsilon$ at $\epsilon=0$. Then

$$
{ }_{1} I\left(\tau, x^{j}\right)=\lim _{\epsilon \rightarrow 0} \frac{d}{d \epsilon} \int_{0}^{\tau / \epsilon} \int_{4 \pi} f\left(\tau-\epsilon r, y^{j}, \epsilon\right) d \Omega_{y} d r
$$

exists provided $\left(f_{, \epsilon}-r f, \tau\right)(\tau-\epsilon r, \ldots)$ is integrable over $C$ as $\epsilon \rightarrow 0$. Then we have

$$
{ }_{1} I\left(\tau, x^{j}\right)=\lim _{\epsilon \rightarrow 0}\left[\int_{0}^{\tau / \epsilon} \int_{4 \pi}\left(f_{, \epsilon}-r f, \tau\right)\left(\tau-\epsilon r, y^{j}, 0\right) d r d \Omega_{y}-\tau \epsilon^{-2} \int_{4 \pi} f\left(0, y^{j}, 0\right) d \Omega_{y}\right] .
$$

Proof. The angular integral multiplied by $\epsilon^{-2}$ in (4.14) exists by the hypotheses, and further derivatives of it may be taken by Lemma 1 . We do not have a general proof that $f_{, \epsilon}-r f_{, \tau}$ will be integrable for the $f$ 's we encounter, so one can only proceed step-by-step, algorithmically showing that each order of differentiation is finite. We have again used assumption (iii) of Sec. III. This completes the proof of the lemma.

We now return to the proof of the post-Newtonian hierarchy theorem. Since the initial data imply ${ }_{n} T^{\tau \tau},{ }_{n} T^{\tau i}$, ${ }_{n} T^{i j}$ all vanish for $n \leq 3$ at $\tau=0$, the initial-value constraints also give that all the data for ${ }_{n} \bar{h}^{\mu \nu}$ likewise vanish for $n \leq 3$. Because the evolution equations for each order are linear, with inhomogeneous terms involving only lower orders, they imply that for $\tau>0$ the solution for ${ }_{n} \bar{h}^{\mu \nu}$ and ${ }_{n} T^{\mu \nu}$ will vanish for $n \leq 3$. Therefore, the first interesting dynamical effects occur at $n=4$. However, first we ought to inquire about the geometry of the limiting manifold, $\epsilon=0$ of our dynamical-time map. We have a degenerate contravariant metric: $\eta^{\tau \tau}=\epsilon^{2} \eta^{00} \rightarrow 0$ while $\eta^{i j}=\delta^{i j}$, unaffected by $\epsilon$. Similarly, the covariant metric is singular, $\eta_{\tau \tau} \rightarrow \infty$. On the other hand, the Christoffel symbols are well behaved: to lowest order

$$
\Gamma_{\tau \tau}^{i}=-\frac{1}{2} \bar{h}_{\tau \tau}{ }^{i}+\frac{1}{4} \bar{h}^{\alpha}{ }_{\alpha}{ }^{i} \eta_{\tau \tau} \rightarrow-\frac{1}{4} \nabla_{4}^{i} \bar{h}^{\tau \tau},
$$

which turns out to be the Newtonian connection, since we shall shortly see that $-{ }_{4} \bar{h}^{\tau \tau} / 4$ is the Newtonian potential. See Misner, Thorne, and Wheeler ${ }^{16}$ and Ehlers ${ }^{23}$ for discussions of this Newtonian spacetime.

At fourth order the initial-value equations give us

$$
{ }_{4} \bar{h}^{\mu \tau}\left(\tau=0, x^{j}\right)=-16 \pi \nabla^{-2}\left[{ }_{4} T^{\mu \tau}(\tau=0)\right],
$$

where we use the shorthand

$$
\nabla^{-2}[f]\left(x^{j}\right)=-\frac{1}{4 \pi} \int f\left(y^{j}\right) r^{-1} d^{3} y .
$$

For $\tau \neq 0$ we determine ${ }_{4} \bar{h}^{\mu \nu}$ from Eq. (4.3). Consider first ${ }_{4} \bar{h}^{\tau \tau}$. The integrals over $S$ make no contribution to ${ }_{4} \bar{h}^{\tau \tau}$ since ${ }_{4} T^{\tau \tau}(\tau=0)$ is of compact spatial support. ${ }^{13}$ The integral over $C$ gives (since the integrand is of compact spatial support)

$$
{ }_{4} \bar{h}^{\tau \tau}\left(\tau, x^{j}\right)=4 \int_{\text {all } y^{j}} \frac{{ }_{2} \rho\left(\tau, y^{j}\right)}{r} d^{3} y
$$


which is -4 times the Newtonian potential of ${ }_{2} \rho$. (This confirms our earlier conclusion about the Christoffel symbol.) For $\bar{h}^{\text {ri }}$ the same reasoning leads to

$$
{ }_{4} \bar{h}^{\tau i}\left(\tau, x^{j}\right)=4 \int_{\text {all } y^{j}} \frac{2 \rho\left(\tau, y^{j}\right){ }_{1} v^{i}\left(\tau, y^{j}\right)}{r} d^{3} y .
$$

For $\bar{h}^{i j}$ the calculation is similar, but there are two contributions to

$$
{ }_{4} \Lambda^{i j}{ }_{4} T^{i j}={ }_{2} \rho_{1} v_{1}^{i} v^{j}+{ }_{4} \rho \eta^{i j}
$$

and

$$
{ }_{4} t_{\mathrm{LL}}^{i j}=(1 / 64 \pi)\left({ }_{4} \bar{h}^{\tau \tau, i}{ }_{4} \bar{h}^{\tau \tau, j}-\frac{1}{2} \eta^{i j}{ }_{4} \bar{h}^{\tau \tau, k}{ }_{4} \bar{h}^{\tau \tau}{ }_{, k}\right) .
$$

The first contribution is of compact support, and the second satisfies the condition of Lemma 2(ii), since [using Eq. (4.21) below]

$$
\begin{aligned}
{ }_{4} \bar{h}^{\tau \tau}, \tau=4 \int{ }_{2} \rho_{, \tau} r^{-1} d^{3} y & =-4 \int\left({ }_{2} \rho_{1} v^{j}\right)_{, j} r^{-1} d^{3} y \\
& =-4 \int{ }_{2} \rho_{1} v^{j} y_{j} r^{-3} d^{3} y .
\end{aligned}
$$

Outside some radius $R,{ }_{4} \bar{h}^{\tau \tau}$ falls off as $r^{-1}$ while ${ }_{4} \bar{h}^{\tau \tau}$, is bounded by $\mathrm{Ar}^{-3}$ from some constant $A$. We therefore do not need retardation in this, the Newtonian approximation.

We thus get

$$
{ }_{4} \bar{h}^{i j}\left(\tau, x^{k}\right)=4 \int_{\text {all } y^{k}} \frac{{ }_{4} T^{i j}\left(\tau, y^{k}\right)+{ }_{4} t_{\mathrm{LL}}^{i j}\left(\tau, y^{k}\right)}{r} d^{3} y .
$$

The $\tau$ component of the conservation law (3.9) also has its first nonvanishing derivative at this order, which is

$$
\left.{ }_{2} \rho\left(\tau, y^{j}\right)_{, \tau}+{ }_{2} \rho\left(\tau, y^{j}\right)_{1} v^{i}\left(\tau, y^{j}\right)\right)_{i}=0 .
$$

Similarly, the $i$ component is obtained from the above expression for ${ }_{4} \Lambda^{i j}$ :

$$
\left({ }_{2} \rho_{1} v^{i}\right)_{, \tau}+\left({ }_{2} \rho_{1} v^{i}{ }_{1} v^{j}\right)_{, j}+{ }_{4} p^{, i}-{ }_{2} \rho_{4} \bar{h}^{\tau \tau, i} / 4=0 .
$$

This, in view of Eqs. (4.18) and (4.21), is equivalent to Euler's equation. So all the equations of Newton's theory come out at fourth order in this approach. This is in contrast to the usual ordering, where the Newtonian approximation uses terms of second, third, and fourth order. ${ }^{14}$

Our expressions for, say ${ }_{2} \rho$ and ${ }_{4} \bar{h}^{\tau \tau}$, are the limits for $\epsilon \rightarrow 0$ of the derivatives $\frac{1}{2} \partial^{2} \rho / \partial \epsilon^{2}$ and $(1 / 4 !) \partial^{4} \bar{h}^{\tau \tau} / \partial \epsilon^{4}$. By assumption (ii) of Sec. III these limits exist. From the existence of the next order's derivatives as well, which we establish below, we conclude that a solution $\left\{{ }_{2} \rho,{ }_{1} v^{i},{ }_{4} \rho\right\}$ of Newton's equations is in an asymptotic approximation to a regular, asymptotically Newtonian sequence $\left\{T^{\alpha \beta}, \bar{h}^{\alpha \beta}\right\}$ in the sense that when Eqs. (4.18)-(4.22) are satisfied,

$$
\begin{aligned}
& { }_{6} \Lambda^{\tau \tau}=-\frac{7}{128 \pi}{ }_{4} \bar{h}^{\tau \tau}, k{ }_{4} \bar{h}^{\tau \tau, k}+{ }_{4} \rho+{ }_{2} \rho\left({ }_{1} v^{k}{ }_{1} v_{k}+\frac{3}{2}{ }_{4} \bar{h}^{\tau \tau}\right) \text {, } \\
& { }_{6} \Lambda^{\tau i}=\frac{1}{8 \pi}\left({ }_{4} \bar{h}^{\tau \tau}{ }_{, k} \bar{h}^{\tau[k, i]}-\frac{3}{8}{ }_{4} \bar{h}^{\tau \tau, i}{ }_{4} \bar{h}^{\tau k}{ }_{, k}\right)+{ }_{4} \rho_{1} v^{i}+{ }_{2} \rho_{3} v^{i}+{ }_{2} \rho_{1} v^{i}\left({ }_{1} v^{k}{ }_{1} v_{k}+\frac{3}{2}{ }_{4} \bar{h}^{\tau \tau}+{ }_{4} p /{ }_{2} \rho\right) \text {, } \\
& \left.{ }_{6} \Lambda^{i j}=\frac{1}{16 \pi}\left[4{ }_{4} \bar{h}^{\tau[i, k]}{ }_{4} \bar{h}_{[k}^{\tau}, j\right]-4{ }_{4} \bar{h}^{\tau \tau,(i}{ }_{4} \bar{h}^{j) k}{ }_{, k}+\frac{1}{2}{ }_{4} \bar{h}^{\tau \tau,(i}{ }_{4} \bar{h}^{|k|}{ }_{k}, j\right)+\frac{1}{2}{ }_{4} \bar{h}^{\tau \tau,(i}{ }_{6} \bar{h}^{|\tau \tau|, j)} \\
& \left.-\frac{1}{2}{ }_{4} \bar{h}^{\tau \tau}{ }_{4} \bar{h}^{\tau \tau,(i}{ }_{4} \bar{h}^{|\tau \tau|, j)}+{ }_{4} \bar{h}^{\tau k, l}{ }_{4} \bar{h}_{[k, l]}^{\tau} \eta^{i j}-\frac{3}{8}\left({ }_{4} \bar{h}^{\tau k}{ }_{, k}\right)^{2} \eta^{i j}+{ }_{4} \bar{h}^{\tau \tau}{ }_{, k}{ }_{4} \bar{h}^{k l}{ }_{, l} \eta^{i j}\right] \\
& +{ }_{4} \rho_{1} v^{i}{ }_{1} v^{j}+2{ }_{2} \rho_{1} v^{(i}{ }_{3} v^{j)}+{ }_{6} p \eta^{i j}+{ }_{2} \rho_{1} v^{i}{ }_{1} v^{j}\left({ }_{1} v^{k}{ }_{1} v_{k}+\frac{3}{2}{ }_{4} h^{\tau \tau}+{ }_{4} p /{ }_{2} \rho\right)+\frac{1}{2}{ }_{4} p{ }_{4} h^{\tau \tau} \eta^{i j} \text {. }
\end{aligned}
$$
$p=\epsilon^{4}{ }_{4} p$, and $u^{i} / u^{0}=\epsilon_{1} v^{i}$. ishes. Therefore, we have

$$
{ }_{3} \rho={ }_{2} v^{i}={ }_{5} \bar{h}^{\mu \tau}=0 .
$$
have therefore

$$
{ }_{5} \bar{h}^{i j}=-4 \int{ }_{4} \Lambda^{i j}{ }_{\tau}\left(\tau, y^{k}\right) d^{3} y .
$$

for any fixed $\tau, 0<\tau<\tau_{1}$ provided that at $\tau=0, \rho=\epsilon_{2}^{2} \rho$,

At the next order, fifth order in $\epsilon$, the constraint equations (3.12) are easily shown to imply that ${ }_{5} \bar{h}^{\mu \tau}=0$ at $\tau=0$, so the integrands for the initial-data integrals for $\bar{h}^{\mu \nu}$ in Eq. (4.3) vanish at this order. The retarded-integral part of $\bar{h}^{\mu \tau}$ also vanishes at this order, for a variety of reasons. It is related to the fourth-order integral by Eq. (4.14) of Lemma 3. The three pieces are treated separately: The surface integral vanishes because ${ }_{4} \Lambda^{\mu v}=O\left(r^{-4}\right)$, the integral over ${ }_{4} \Lambda^{\mu \tau}{ }_{, \tau}$ is zero because of the conservation law ${ }_{4} \Lambda^{\mu \tau}{ }_{, \tau}=-{ }_{4} \Lambda^{\mu j}{ }_{j}$, and in the remaining piece ${ }_{5} t_{\mathrm{LL}}^{\mu \nu}$ van-

$$
{ }_{5} \bar{h}^{\mu \tau}=4 \int_{\text {all } y^{k}} r^{-1}{ }_{5} T^{\mu \tau}\left(\tau, y^{k}\right) d^{3} y .
$$

The equations of motion follow from (3.9), and may be taken to determine ${ }_{3} \rho$ and ${ }_{2} v^{i}$. But these functions determine ${ }_{5} T^{\mu \nu}$ as well, so a self-consistent solution for our zero initial data for ${ }_{3} \rho$ and ${ }_{2} v^{i}$ is that they all vanish:

For ${ }_{5} \bar{h}^{i j}$ the situation is different, because there is no conservation law to wipe out the integral over ${ }_{4} \Lambda_{, \tau}^{i j}$. We

We can ignore the retardation here because ${ }_{4} \Lambda^{i j}$ satisfies the conditions of Lemma 2(ii), essentially because ${ }_{4} \bar{h}^{\tau \tau}$ does, as we showed earlier. So at $\frac{1}{2}-P N$ order there is one nonzero contribution, which is a function only of $\tau$ and which turns out to contribute to radiation reaction. The existence of all the limits at this order guarantees the asymptotic nature of the Newtonian approximation.

At $\epsilon^{6}$ order, where the post-Newtonian approximation turns up, we obtain the following metric:

$$
\begin{aligned}
{ }_{6} \bar{h}^{\mu \nu}\left(\tau, x^{k}\right)= & 4 \int_{6} \Lambda^{\mu \nu}\left(\tau, y^{k}\right) r^{-1} d^{3} y \\
& +2 \int_{4} \Lambda^{\mu \nu}{ }_{, \tau \tau}\left(\tau, y^{k}\right) r d^{3} y .
\end{aligned}
$$

The first term is obvious; the second contains retardation effects from fourth order. The integrals in (4.26) are not retarded, and there are no contributions from the initialdata integrals in (4.3) at this order (paper II).

We know ${ }_{4} \Lambda^{\mu \nu}$ from the Newtonian step: ${ }_{4} \Lambda^{\tau \tau}={ }_{2} \rho$, ${ }_{4} \Lambda^{\tau i}={ }_{2} \rho_{1} v^{i}, \quad{ }_{4} \Lambda^{i j}={ }_{2} \rho_{1} v^{i}{ }_{1} v^{j}+{ }_{4} p \eta^{i j}+{ }_{4} t^{i j} \mathrm{~L}$ [see after Eq. (4.19) above]. The new source terms are 
Note that to compute ${ }_{6} \Lambda^{i j}$, which will enter the first postNewtonian equation of motion, one needs to compute ${ }_{6} \bar{h}^{\tau \tau}$ using Eq. (4.26) with ${ }_{6} \Lambda^{\tau \tau}$ as source. This happens at each order: The equations to be solved simultaneously determine the motions and fields. The dynamical equations are just

$$
{ }_{6} \Lambda^{\tau \tau}{ }_{, \tau}+{ }_{6} \Lambda^{\tau i}{ }_{, i}=0, \quad{ }_{6} \Lambda^{\tau i}{ }_{, \tau}+{ }_{6} \Lambda^{i j}{ }_{j}=0 .
$$

They determine the new variables at this order: ${ }_{4} \rho,{ }_{3} v^{i}$, and associated fields. Apart from the explicit separation of $\rho$ into its various orders ${ }_{2} \rho,{ }_{4} \rho$, etc., and similarly for $v^{i}$ and $p$, these equations are equivalent to those in Kerlick. ${ }^{10}$

In Eq. (4.26) we neglected retardation, but the terms involving $\bar{h}^{j k}$ in ${ }_{6} \Lambda^{i j}$ above and many of the terms in ${ }_{4} \Lambda^{i j}{ }_{, \tau \tau}$ do not satisfy condition (ii) of Lemma 2 . It turns out that retardation can be neglected anyway, but we have reserved a discussion of this point to paper II, where similar terms at higher orders will be examined.

Again, these equations govern the $\epsilon \rightarrow 0$ limits of the various fields, and as we assume that these equations are well-posed initial-value equations, for which we have here given the initial data ${ }_{4} \rho={ }_{3} v^{i}=\bar{h}^{i j}=0$, it follows that these limits exist. It is easy to check also that the derivatives at the next order exist as well. Equations (4.26)-(4.28) constitute the first post-Newtonian corrections. Taken together with Eq. (4.25) and the Newtonian equations, it gives an asymptotic approximation of order $\epsilon^{6}$ to the regular, asymptotically Newtonian sequence:

$$
\begin{aligned}
& \qquad \bar{h}^{\tau \tau}\left(\tau, x^{j}, \epsilon\right)=\epsilon^{4}{ }_{4} \bar{h}^{\tau \tau}\left(\tau, x^{j}\right)+\epsilon^{6}{ }_{6} \bar{h}^{\tau \tau}\left(\tau, x^{j}\right)+O\left(\epsilon^{7}\right), \\
& \text { etc. } \\
& \text { This completes the proof of our theorem. }
\end{aligned}
$$

\section{DISCUSSION}

We have shown that the Newtonian and postNewtonian approximations derived by previous authors in a formal iteration on $\epsilon$ (or on $c^{-1}$ ) are in fact derivatives with respect to $\epsilon$ of a sequence of fully relativistic solutions, and therefore asymptotic approximations to the sequence. This proof may be extended to higher orders in a straightforward way. At each step one should, following Kerlick, ${ }^{10,11}$ "reduce" the equations by using information about lower-order terms from previous steps. We did this, for example, twice at order $\epsilon^{5}$ : once when we used the fourth-order conservation law ${ }_{4} \Lambda^{\mu v}{ }_{, v}$ to eliminate some terms and once when we showed that retardation can be neglected in (4.25). Up to order $\epsilon^{9}$, the resulting equations are essentially the same ${ }^{24}$ as one obtains by the formal slow-motion iteration on $\epsilon$, but after that point paper II will show that the formal iterates no longer give the actual derivatives of the relativistic solution, because of the above-mentioned retardation problem. Carried to high enough order, our scheme shows that the asymptotic approximation must contain terms of the type $\epsilon^{n} \ln \epsilon$, which were also found by Anderson et al..$^{25}$ in the matchedasymptotic expansion approach. The formal slow-motion iteration assumes that all terms in the approximation will be powers of $\epsilon$, so at some point it inevitably finds logarithmically divergent coefficients of these powers. Kerlick's logarithmic divergences become, in our approach, merely finite coefficients of the functions $\epsilon^{n} \ln \epsilon$ in the asymptotic approximation.

It follows that the approximations generated by Ker- lick, ${ }^{10,11}$ Anderson and Decanio, ${ }^{14}$ and Chandrasekhar et $a l .^{2-4}$ are also asymptotic, provided they are treated as Kerlick treats them. But here one must be cautious, since none of these authors separates out different orders in $\rho, p$, and $v^{i}$. Therefore, there are two interpretations one can put on their formalisms. One is to say that, at postNewtonian order, say, the formal function $\rho$ is to be identified with $\epsilon^{2}{ }_{2} \rho+\epsilon^{4}{ }_{4} \rho$, so that Kerlick's function $U=\int \rho r^{-1} d^{3} y$, which looks like the Newtonian potential, is actually more complex. (One could in fact be less charitable and take $\rho$ to be the full $\rho$ with all orders involved, in which case even the older definition of the Newtonian approximation contains terms that the present formulation assigns to higher orders. It would still be asymptotic, of course.) The second interpretation is perhaps closer to the view the previous authors had, though they did not make it explicit. That is to ignore the relativistic sequence and to regard the various approximations as terms in a power series in $\epsilon$ (or $c^{-1}$ ) which one hopes converges to a particular relativistic solution for $\epsilon=1$, whose density is $\rho$. Then $\rho$ has no $\epsilon$ dependence, but at the same time the sequence of metrics to which the various approximate $\bar{h}^{\mu \nu}$ s $s$ is asymptotic is not a sequence of solutions of Einstein's equations except at $\epsilon=1$. This view is unsatisfactory, not least of all because the remainder terms, which one in principle wants to know in order to judge one's accuracy, are not the differences between the approximations and exact solutions, but rather between the approximations and an artificial sequence, only one of whose members is a solution.

It is important to note that the limits $\epsilon \rightarrow 0$ and $\tau \rightarrow 0$ in our sequence do not necessarily commute (J. Ehlers, private communication). In this paper we have always taken $\epsilon$ to zero before $\tau$, thereby showing that the postNewtonian hierarchy is asymptotic at fixed $\tau>0$. The only place we set $\tau=0$ is in the surface integrals at $t=0$ in Kirchhoff's formula, which is used for $\epsilon \neq 0$. In fact, for certain functions, such as, ${ }_{4} \bar{h}^{\tau \tau}$ the limits commute, but for ${ }_{4} \bar{h}^{i j}$ they do not. This is because for our initial data some functions that are nonzero for large $t$ become zero as $t$ decreases into the domain of dependence of the vacuum region of the initial hypersurface. This does not affect the asymptotic convergence of the post-Newtonian equations for $\tau>0$, since this region shrinks to zero in $\tau$ time.

Is our approach to the problem unique? Certainly not. There are many ways one can vary our definitions and expect a satisfactory result. It may of course be possible to abandon the initial-value viewpoint in favor of some asymptotic wave condition, but this is not clear. But within the initial-value approach many variants are possible.

(1) The coordinate maps involved (charts $t, x^{j}$ and the definition $\tau=\epsilon t$ ) are unique (modulo Lorentz transformations) only in the $\epsilon \rightarrow 0$ limit, where we have flat space and a Lorentz coordinate system. Any variations that vanish in this limit will turn up as changes of gauge in the various orders of approximation.

(2) There is nothing unique about $\epsilon$. If we defined another parameter $\mu=\epsilon+\epsilon^{2}$, the the $\mu$ Newtonian approximation would be the same as the $\epsilon$ Newtonian one, but higher-order corrections would differ in the two schemes. There can be no unique definition of a post-Newtonian correction unless the Newtonian quantity vanishes. (This 
was emphasized in another context by Stewart and Walker. $^{26}$ ) The full approximation (Newtonian plus postNewtonian) is, of course, invariant.

(3) Our choice of initial data was convenient but not necessary. We could also have allowed more freedom in $\bar{h}^{i j}$, as paper II will discuss.

Such variants deserve more study, because the present definition can lead to the following curious circumstance. Suppose we wish to approximate a relativistic neutron star in small-amplitude nonradial pulsation by a Newtonian one, whose pulsations are easier to study. We must fit the relativistic solution into a sequence, each member of which at $t=0$ has the same (scaled) density distribution. But as $\epsilon \rightarrow 0$ the initial density distribution becomes farther and farther from an equilibrium from that $\epsilon$, so that time dependence of $\epsilon \neq 1$ solutions may be dominated by this spherical disequilibrium, rather than by the small nonradial pulsation. The usefulness of the Newtonian approximation is not clear in this circumstance. This "paradox" arises because, although for general initial data the velocities that result will be of order (typical radius)/(dynamical time), and therefore scale with $\epsilon$, in this particular example there is a cancellation of gravity and pressure for one member of the sequence which allows it to have unusually small velocities. This condition of equilibrium does not scale with the initial data for all orders in $\epsilon$. This question is important in view of the demonstration by Balbinski and Schut ${ }^{27}$ that the quadrupole radiation-reaction formula may not estimate damping times of nonradially pulsating neutron stars well.

A related question is whether the post-Newtonian hierarchy can be applied to the binary pulsar, which is generally regarded as a test of the radiation-reaction predictions of general relativity. ${ }^{8}$ Paper II will discuss this in detail, but the matter of principle which we raise here is the strong internal gravity of these stars. We subscribe to what seems to be the general view, that because of the equivalence principle the internal structure of the stars is irrelevant to the gravitational radiation emitted by their orbital motion, which is governed by weak gravitational fields. ${ }^{28}$ But only a detailed examination of the remainder term in the asymptotic expansion can answer the question within this framework. The difficulty of such a task may be enormous because the remainder term involves the relativistic solutions for $\epsilon \neq 0$, not just their $\epsilon=0$ limits.

\section{ACKNOWLEDGMENTS}

The presentation and substance of this paper have benefited from a number of careful and helpful comments from D. Christodoulou, J. Ehlers, B. Schmidt, and M. Walker. B.F.S. gratefully acknowledges the support and hospitality of the Max Planck Institute in Garching during part of the period this work was developing.

\section{APPENDIX: NEGLECTING RETARDATION}

Lemma 2 gives a sufficient condition for neglecting retardation, but it still proves possible to neglect retardation when condition (ii) of Lemma 2 does not apply. Here we shall show how this can happen. Equation (4.9) can be written as

$$
I\left(\tau, x^{j}, 0\right)=\lim _{\epsilon \rightarrow 0} \int_{0}^{\tau / \epsilon} \int_{4 \pi} f\left(\tau-r, x^{j}+r n^{j}, 0\right) d r d \Omega_{n}
$$

As in Lemma 2 we suppress the dependence of $f$ on $x^{j}$ and $n^{j}$ and write

$$
f\left(\tau-\epsilon r, x^{j}+r n^{j}, 0\right)=h(\tau-\epsilon r, r) .
$$

Then Eq. (4.11) becomes

$$
\begin{aligned}
\int_{0}^{\tau / \epsilon} h(\tau-\epsilon r, r) d r= & \int_{0}^{\tau / \epsilon} h(\tau, r) d r \\
& -\int_{0}^{\tau / \epsilon} \int_{0}^{\epsilon r} h_{, \tau}(\tau-\eta, r) d \eta d r
\end{aligned}
$$

We can therefore identify the "instantaneous" and "retardation" parts of $I, I_{i}$, and $I_{r}$, where

$$
\begin{aligned}
I_{i} & =\int_{0}^{\tau / \epsilon} h(\tau, r) d r \\
I_{r} & =\int_{0}^{\tau / \epsilon} \int_{0}^{\epsilon r} h_{, \tau}(\tau-\eta, r) d \eta d r \\
& =\epsilon \int_{0}^{\tau / \epsilon} r h_{, \tau}\left(\tau-\eta_{0}, r\right) d r, 0 \leq \eta_{0} \leq \epsilon r
\end{aligned}
$$

and where we have used the mean-value theorem to reduce $I_{r}$, and the angular integration is understood.

We conjecture that $\left|I_{r} / I_{i}\right| \rightarrow 0$ as $\epsilon \rightarrow 0$ if $I_{r}$ exists in the limit. This enables us to neglect retardation. We have no proof of this (one difficulty being the dependence of $\eta_{0}$ on $\epsilon$ and $r$ ), but offer the following examples to show that it is likely to be true for most cases.

Clearly, if the integral in (A4) exists as $\epsilon \rightarrow 0$, then the factor of $\epsilon$ in front of it makes $I_{r}$ vanish, and retardation is negligible. This happens under condition (ii) of Lemma 2.

Suppose, however, that the radial integral in $I_{r}$ diverges but $I_{r}$ remains finite. This will happen if, for example,

$$
\begin{aligned}
& \left|r h_{, \tau}(\tau, r)\right|<A, \\
& h(\tau, r)=N(\tau) / r+O\left(r^{-2}\right) \text { as } r \rightarrow \infty,
\end{aligned}
$$

$h(r)$ is regular at $r=0$,

where $A$ is a constant and $N(\tau)$ is an arbitrary bounded function of $\tau$. The second condition is only asymptotic. Then we have for sufficiently small $\epsilon$

$$
I_{i}=N(\tau) \ln (\tau / \epsilon)+O\left(\epsilon^{0}\right), \quad\left|I_{r}\right|<A
$$

In this case $\left|I_{r} / I_{i}\right| \rightarrow 0$ so we can still neglect retardation. It is true that $I_{i}$ diverges, which would mean that the sequence of solutions is not differentiable with respect to $\epsilon$ at the order at $\epsilon=0$. But this does not prevent one from developing asymptotic approximations near $\epsilon=0$ : $\sin \epsilon \ln \epsilon$ is not differentiable at $\epsilon=0$ but $\epsilon \ln \epsilon$ is asymptotic to it.

If we consider more divergent functions, like 


$$
\begin{aligned}
& \left|r h_{, \tau}(\tau, r)\right|<A r, \\
& h(\tau, r)=N(\tau)+O\left(r^{-1}\right) \text { as } r \rightarrow \infty, \\
& h(\tau, r) \text { regular at } r=0 .
\end{aligned}
$$

then we have

$$
I_{i}=\tau N(\tau) / \epsilon+O(\ln \epsilon), \quad\left|I_{r}\right|<A \tau^{2} / 2 \epsilon .
$$

In this case $I_{r}$ is not negligible next to $I_{i}$, and it is divergent. This happens as well if we take higher powers of $r$ in (A7). It seems likely that if $I_{i}$ diverge then $I_{i} / I_{r}$ remain finite.
${ }^{*}$ Permanent address.

${ }^{1}$ A. Einstein, Ann. Phys. (Leipzig) 49 (1916).

${ }^{2}$ S. Chandrasekhar, Astrophys. J. 142, 1488 (1965).

${ }^{3}$ S. Chandrasekhar and Y. Nutku, Astrophys. J. 158, 55 (1969).

${ }^{4}$ S. Chandrasekhar and E. P. Esposito, Astrophys. J. 160, 153 (1970).

${ }^{5}$ R. Blandford and S. A. Teukolsky, Astrophys. J. 205, 580 (1976) and R. Epstein, ibid. 216, 92 (1977).

${ }^{6}$ See C. M. Will, in General Relativity-An Einstein Centenary Survey, edited by S. W. Hawking and W. Israel (Cambridge University Press, New York, 1979).

${ }^{7}$ J. Ehlers, A. Rosenblum, J. N. Goldberg, and P. Havas, Astrophys. J. 208, L77 (1976).

${ }^{8}$ J. H. Taylor, L. A. Fowler, and P. M. McCulloch, Nature 277, 439 (1979).

${ }^{9}$ J. Ehlers, report (unpublished).

${ }^{10}$ G. D. Kerlick, Gen. Relativ. Gravit. 12, 467 (1980).

${ }^{11}$ G. D. Kerlick, Gen. Relativ. Gravit. 12, 521 (1980).

${ }^{12}$ M. Walker and C. M. Will, Phys. Rev. Lett. 45, 1741 (1980).

${ }^{13}$ B. F. Schutz, Phys. Rev. D 22, 249 (1980).

14J. L. Anderson and T. C. Decanio, Gen. Relativ. Gravit. 6, 197 (1975).

${ }^{15}$ S. Chandrasekhar, Stellar Structure (University of Chicago Press, Chicago, 1939).

${ }^{16}$ Our notation and conventions follow C. W. Misner, K. S. Thorne, and J. A. Wheeler, Gravitation (Freeman, San Francisco, 1973). In particular, Greek indices run from 0 to 3, Latin from 1 to 3 .

${ }^{17}$ L. D. Landau and E. M. Lifshitz, Classical Theory of Fields (Addison-Wesley, Reading, Massachusetss, 1971).

${ }^{18}$ T. Futamase, Ph.D. thesis, University College, Cardiff 1981 (unpublished).

${ }^{19}$ N. Ó Murchadha and J. W. York, Jr., Phys. Rev. D $\underline{10}, 2345$
(1974).

${ }^{20}$ It is not really necessary that each spacetime be diffeomorphic to $R^{4}$, but only that the region illustrated in Fig. 1, bounded by the hypersurfaces $t=0$ and $t=\tau_{1} / \epsilon$ for some fixed $\tau_{1}$, be diffeomorphic to $R^{3} x(0,1)$ for each $\epsilon$.

${ }^{21}$ We take the point of view that $h^{\mu v}$ is a tensor, defined by giving its components in the assumed de Donder coordinate system as the difference between the tensor density $\sqrt{-g} g^{\mu \nu}$ and the matrix $\eta^{\mu \nu}$. Since the transformation $t \rightarrow \tau$ changes the coordinates, $h^{\tau \tau}$ is not given by $\sqrt{-g} g^{\tau \tau}-\eta^{\tau \tau}$ in the new coordinate system, but rather by the tensor transformations from the old one.

${ }^{22}$ F. G. Friedlander, The Wave Equation on a Curved Spacetime (Cambridge University Press, New York, 1975).

23J. Ehlers, report (unpublished).

${ }^{24}$ We say "essentially" because there is one term in $h^{\tau \tau}$ at second post-Newtonian order which arises from the homogeneous equation, but is independent of position and so does not affect the equation of motion.

25J. L. Anderson, R. E. Kates, L. S. Kegeles, and R. G. Madonna, Phys. Rev. D 25, 2038 (1982).

26J. Stewart and M. Walker, Proc. R. Soc. London A341, 49 (1974).

${ }^{27}$ E. F. L. Balbinski and B. F. Schutz, Mon. Not. R. Astron. Soc. 200, 43P (1982).

${ }^{28}$ Strong support for this view comes from the matchedasymptotic-expansion approach of R. E. Kates, Phys. Rev. D 22, 1871 (1980), and the point-particle methods of T. Damour and N. Deruelle, C. R. Acad. Sci. Ser. B 293, II 5037 (1981); 293, 877 (1981). A variant on the present limit which preserves the strong internal gravity of the stars will be suggested in the following paper. 